\title{
Paschen-Back effect involving atomic fine and hyperfine structure states
}

\author{
K. Sowmya ${ }^{1}$, K. N. Nagendra ${ }^{1}$, M. Sampoorna ${ }^{1}$ and J. O. Stenflo ${ }^{2,3}$ \\ ${ }^{1}$ Indian Institute of Astrophysics, Koramangala, Bengaluru, India \\ email: ksowmya@iiap.res.in; knn@iiap.res.in; sampoorna@iiap.res.in \\ ${ }^{2}$ Institute of Astronomy, ETH Zurich, CH-8093 Zurich, Switzerland \\ ${ }^{3}$ Istituto Ricerche Solari Locarno, Via Patocchi, 6605 Locarno-Monti, Switzerland \\ email: stenflo@astro.phys.ethz.ch
}

\begin{abstract}
The linear polarization in spectral lines produced by coherent scattering is significantly modified by the quantum interference between the atomic states in the presence of a magnetic field. When magnetic fields produce a splitting which is of the order of or greater than the fine or hyperfine structure splittings, we enter the Paschen-Back effect (PBE) regime, in which the magnetic field dependence of the Zeeman splittings and transition amplitudes becomes non-linear. In general, PBE occurs for sufficiently strong fields when the fine structure states are involved and for weak fields in the case of hyperfine structure states. In this work, we apply the recently developed theory of $\mathrm{PBE}$ in the atomic fine and hyperfine structure states including the effects of partial frequency redistribution to the case of Li I $6708 \AA$ doublet. We explore the signatures of $\mathrm{PBE}$ in a single scattering event and their applicability to the solar magnetic field diagnostics.
\end{abstract}

Keywords. atomic processes, polarization, scattering, Sun: magnetic fields

\section{Introduction}

The non-linear effects in the PBE regime that occur when the Zeeman splitting is comparable to the fine or hyperfine structure splittings have previously been exploited for magnetic field diagnostics in the case of molecular lines. With a motivation to explore the diagnostic potential of the PBE in the atomic states, here we try to identify its signatures in the polarized light emitted in a single scattering process of the unpolarized incident radiation off an atom with a finite electron or nuclear spin. In the case of the Sun, the Li I D line system at $6708 \AA$ serves as the best candidate to explore such signatures. This is because both the fine and hyperfine structure states of this line system exhibit PBE for the field strengths encountered in the solar atmosphere. Therefore we apply the partial redistribution scattering theory developed for PBE in fine structure states (Sowmya et al. 2014b) and for PBE in hyperfine structure states (Sowmya et al. 2014a) to study the signatures of PBE in the Li I D line system. We consider both the stable isotopes of $\mathrm{Li}$, namely ${ }^{6} \mathrm{Li}$ and ${ }^{7} \mathrm{Li}$. All the relevant atomic parameters for both the isotopes (including their abundance) are taken from Table 1 of Belluzzi et al. (2009).

The partial frequency redistribution matrices derived in Sowmya et al. (2014a, 2014b) are used for the computations presented in this paper. We consider a right-handed threedimensional Cartesian coordinate system, with the magnetic field along the $z$-axis. An unpolarized beam of radiation is incident along the $x$-axis (i.e., inclination $\theta^{\prime}=90^{\circ}$ and azimuth $\chi^{\prime}=0^{\circ}$ ). The scattered radiation makes an angle $\theta=\beta$ with the magnetic field and has an azimuth $\chi=90^{\circ}$. We consider two cases, namely, a horizontal field parallel to the line of sight $\left(\beta=0^{\circ}\right)$ and a vertical field perpendicular to the line of sight $\left(\beta=90^{\circ}\right)$. Following Belluzzi et al. (2009) the Doppler width is chosen as $60 \mathrm{~m} \AA$ for the spectral 
lines so that the scattered Stokes profiles closely resemble the observations of this line system by Stenflo (2011). Because the lithium lines are optically thin and as only single scattering is considered here, the Stokes profiles computed for the two isotopes are added after weighting them with their percentage abundances, to obtain the actual scattered Stokes profiles from both isotopes together.

\section{PBE in atomic states}

In this section, we describe the behavior of the atomic levels with fine or hyperfine structure splitting, under the influence of a magnetic field, and discuss the scattered Stokes profiles obtained in a single scattering of unpolarized incident radiation.

\subsection{Crossing of atomic states}

As described earlier, the PBE regime is characterized by the crossing of magnetic substates belonging to different fine or hyperfine structure states. In the case of Li atom, we see two level crossings when we consider the magnetic substates belonging to the fine structure states of the two stable isotopes ${ }^{6} \mathrm{Li}$ and ${ }^{7} \mathrm{Li}$ (see Figure $1(\mathrm{a})$ ). These level crossings occur for field strengths in the range $3-5 \mathrm{kG}$. The magnetic substates belonging to hyperfine structure states of ${ }^{6} \mathrm{Li}$ (nuclear spin, $I_{s}=1$ ) show 9 crossings corresponding to magnetic fields in the range $0-4 \mathrm{G}$ (see Figure $1(\mathrm{~b})$ ), while those of ${ }^{7} \mathrm{Li}\left(I_{s}=3 / 2\right)$ show 14 crossings in the range $0-10 \mathrm{G}$ (see Figure $1(\mathrm{c})$ ). Because of the relatively smaller hyperfine structure splitting (in comparison to the fine structure splitting), the fields required to enter the $\mathrm{PBE}$ regime are much smaller in the case of hyperfine structure states as compared to those in the fine structure case.

\subsection{Non-linear splitting}

Another characteristic of the PBE regime is the non-linearity of the magnetic splitting in the presence of a magnetic field. In the linear Zeeman regime, the magnetic components are symmetrically displaced about the line center position while in the PBE regime, they are displaced asymmetrically. This can be clearly seen in Figure 1(d) where we have plotted the strengths of the magnetic components as a function of their displacement from the line center position for the level crossing field strength $(B=3.238 \mathrm{kG})$ in the case of fine structure states. Here the solid lines correspond to the components of ${ }^{7} \mathrm{Li}$ and the dashed lines to those of ${ }^{6} \mathrm{Li}$. The component strengths are multiplied by their respective isotope abundances. Similar line splitting diagrams for the magnetic components resulting from the transitions between the magnetic substates belonging to the hyperfine structure states of $\mathrm{D}_{2}$ lines of ${ }^{6} \mathrm{Li}$ and ${ }^{7} \mathrm{Li}$ are respectively shown in Figure $1(\mathrm{e})$ and Figure $1(\mathrm{f})$. One can again notice the asymmetric splitting of the magnetic components caused by the PBE.

\subsection{Net circular polarization}

In the linear Zeeman regime, because of the symmetric splitting of the $\sigma$-components, the Stokes $V$ profiles exhibit perfect antisymmetry about the line center. As a result the net circular polarization (NCP) defined as $\int V d \lambda$ becomes zero. On the other hand, because of the non-linear splitting of the magnetic components in the PBE regime, the Stokes $V$ profiles are no longer antisymmetric about the line center. Thus the NCP in this case is non-zero. This is illustrated in Figure 2 for the fine structure case in the presence of a horizontal field parallel to the line of $\operatorname{sight}\left(\beta=0^{\circ}\right)$. We notice that the NCP value increases with the increasing field strength, reaches a maximum around the level-crossing field strengths and then decreases as shown in Figure 2. The NCP becomes zero once 

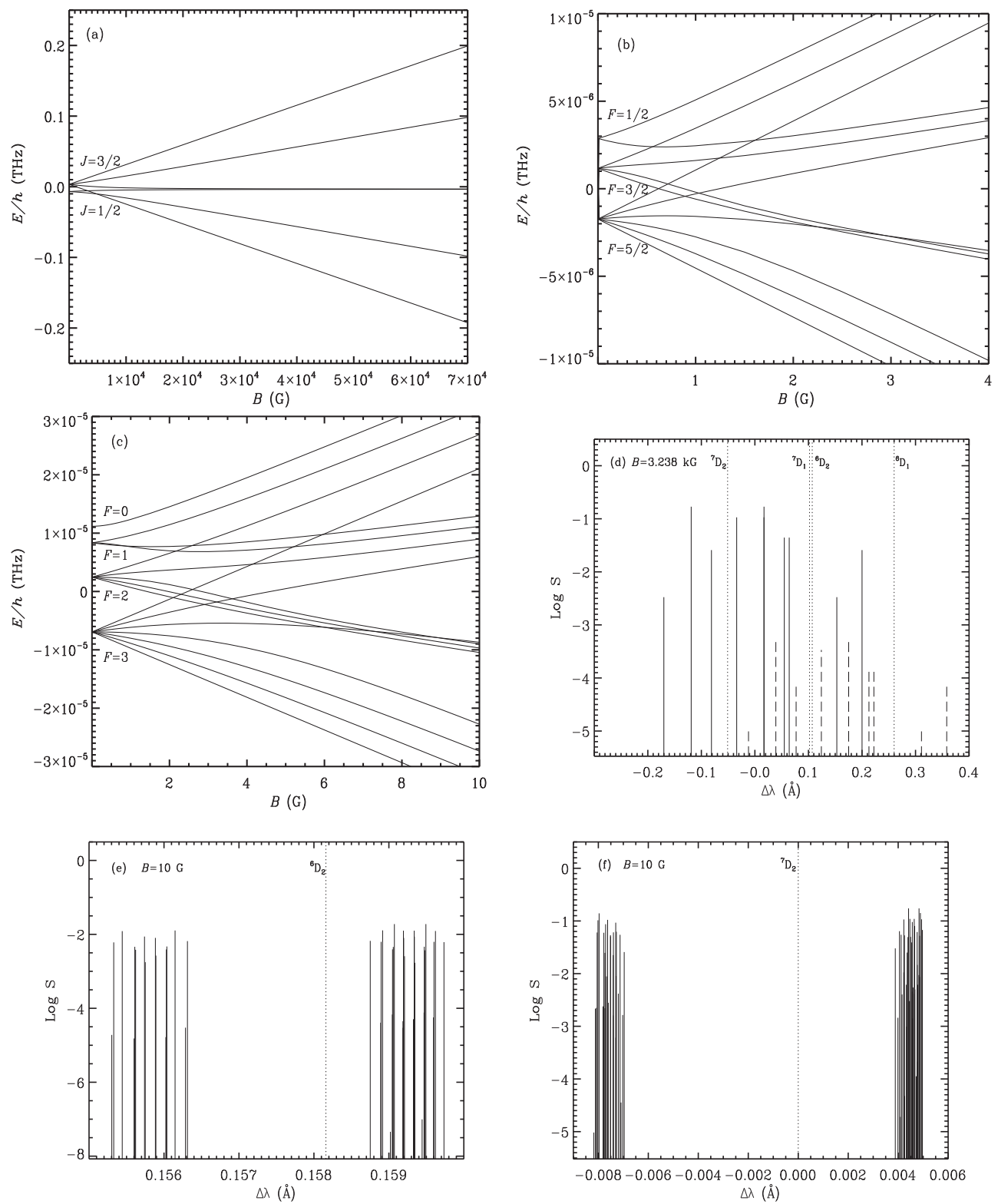

Figure 1. Level-crossing diagrams for (a) the fine structure states of ${ }^{7} \mathrm{Li}$ (note that a similar behavior is exhibited by the fine structure states of ${ }^{6} \mathrm{Li}$, and hence not shown), (b) the hyperfine structure states of ${ }^{6} \mathrm{Li}$, and (c) the hyperfine structure states of ${ }^{7} \mathrm{Li}$. Line splitting diagrams for the transitions from (d) the fine structure states of ${ }^{7} \mathrm{Li}$ (solid lines) and ${ }^{6} \mathrm{Li}$ (dashed lines), (e) the hyperfine structure states of ${ }^{6} \mathrm{Li}$, and (f) the hyperfine structure states of ${ }^{7} \mathrm{Li}$. The vertical dotted lines in panels (d)-(f) mark the positions of $\mathrm{D}_{1}$ and $\mathrm{D}_{2}$ lines of ${ }^{6} \mathrm{Li}$ and ${ }^{7} \mathrm{Li}$ (denoted here as ${ }^{7} \mathrm{D}_{2},{ }^{7} \mathrm{D}_{1}$ and $\left.{ }^{6} \mathrm{D}_{2},{ }^{6} \mathrm{D}_{1}\right)$.

we enter the complete PBE regime, where, as in the case of the linear Zeeman regime, the splitting once again becomes linear (see also Figure 1(a)). In the case of hyperfine structure, NCP is too small to be noticeable, because of the relatively smaller degree of splitting. 


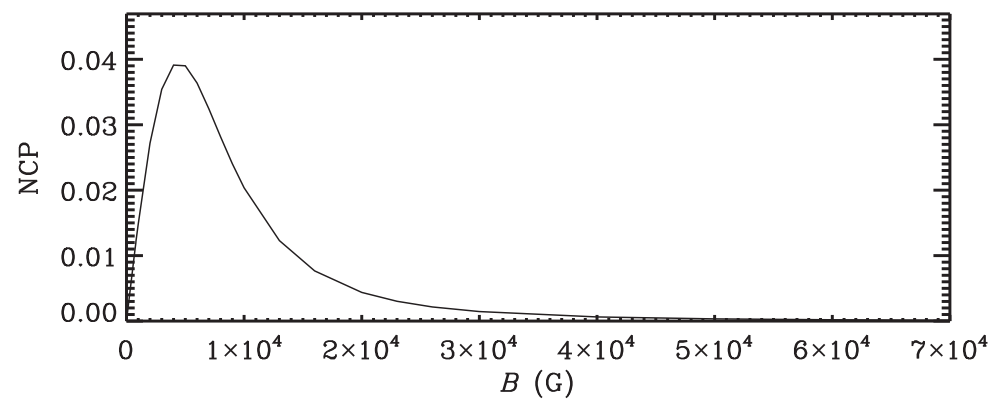

Figure 2. Net Circular Polarization (NCP) for fine structure transitions in the presence of a horizontal field parallel to the line of sight.
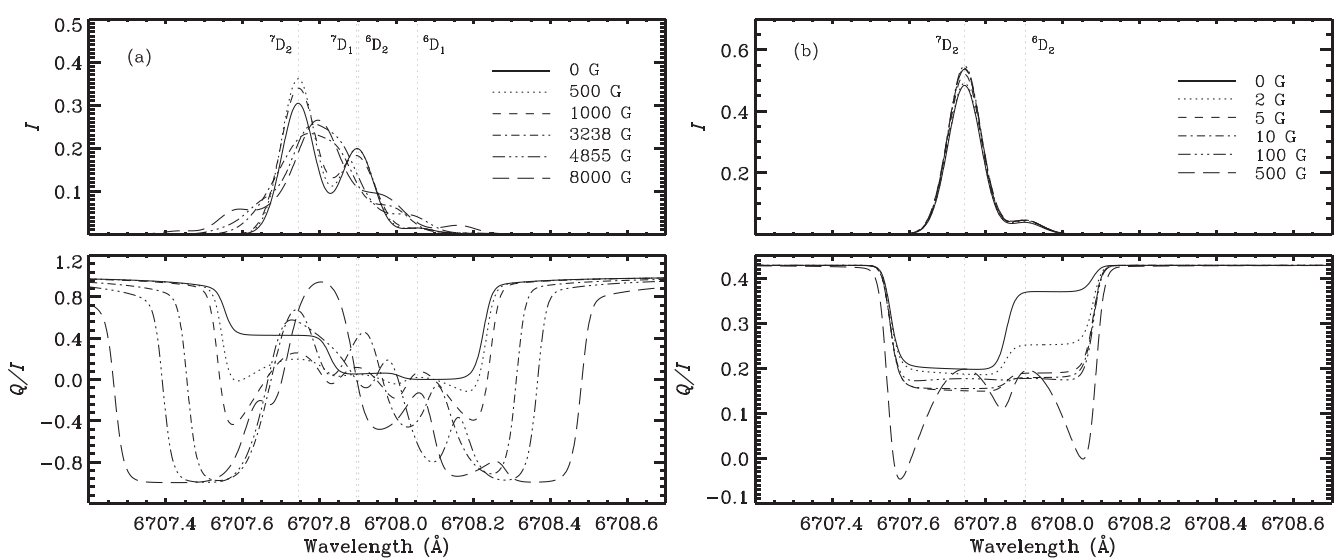

Figure 3. The scattered Stokes profiles in the presence of a vertical magnetic field. Panel (a) shows the fine structure case and panel (b) the hyperfine structure case. The vertical dotted lines mark the positions of the $\mathrm{D}_{1}$ and $\mathrm{D}_{2}$ lines of ${ }^{6} \mathrm{Li}$ and ${ }^{7} \mathrm{Li}$.

\subsection{Effect of a vertical magnetic field}

In Figure 3 we present the Stokes profiles obtained in the presence of a vertical magnetic field $\left(\beta=90^{\circ}\right)$ for (a) the fine structure case involving the $\mathrm{D}_{1}$ and $\mathrm{D}_{2}$ lines of ${ }^{6} \mathrm{Li}$ and ${ }^{7} \mathrm{Li}$ weighted by their respective isotope abundances and (b) the hyperfine structure case involving only the $\mathrm{D}_{2}$ lines of ${ }^{6} \mathrm{Li}$ and ${ }^{7} \mathrm{Li}$ weighted by their respective abundances. The intensity profiles in Figure 3(a) become broader with the increasing field strength because of the increased separation between the magnetic components. This is not the case in Figure 3(b) as the magnetic splittings involved are too small to produce any significant broadening. $Q / I$ decreases as the field strength is increased followed by an increase beyond the value at $B=0$ (see Figure $3(\mathrm{a})$ ), which is attributed to the anti-level crossing effect described in detail in Sowmya et al. (2014a). In Figure 3(b) we only see a decrease in $Q / I$ with the increasing field strength followed by an increase. For stronger fields, signatures of the transverse Zeeman effect are seen. The Stokes $U$ and $V$ are zero owing to the chosen geometry.

\section{Summary}

The PBE occurs when the magnetic energy is comparable to or greater than the energy separation between the unperturbed atomic states. In the incomplete PBE regime, the energy eigenvalues diverge non-linearly with the field strength leading to an asymmetry in 
the splitting produced and crossing of the magnetic substates. The symmetry is retained in the complete PBE regime where the linearity in the behavior of eigenvalues sets in again. In the incomplete PBE regime, the NCP remains non-zero because of the nonlinear splitting. The anti-level crossing effect is seen in the presence of a vertical field because of PBE.

The Stokes profiles presented here are obtained from a single scattering on a Li I atom. A detailed radiative transfer calculation including the scattering redistribution matrices derived in our previous papers would shed more light on the observable signatures of PBE. Modeling of the lines in the Second Solar Spectrum (linearly polarized spectrum of the Sun) with above said theoretical formulation in turn would allow us to explore the diagnostic potential of $\mathrm{PBE}$ in atomic states for solar magnetic fields.

\section{Acknowledgements}

We acknowledge the use of HYDRA cluster facility at the Indian Institute of Astrophysics for computing the results presented in the paper.

\section{References}

Belluzzi, L., Landi Degl'Innocenti, E., \& Trujillo Bueno, J. 2009, ApJ 705, 218

Sowmya, K., Nagendra, K. N., Stenflo, J. O., \& Sampoorna, M. 2014a, ApJ 786, 150

Sowmya, K., Nagendra, K. N., Sampoorna, M., \& Stenflo, J. O. 2014b, ApJ 793, 71

Stenflo, J. O. 2011, in: J. R. Kuhn, D. M. Harrington, H. Lin, S. V. Berdyugina, J. Trujillo

Bueno, S. L. Keil, \& T. Rimmele (eds.), Solar Polarization 6, ASP Conf. Series 437 (San Francisco: ASP), p. 3 